\title{
The dual role of osteopontin in acetaminophen hepatotoxicity
}

\author{
Chun-yan HE H, 2, $^{\text {, Bei-bei LIANG }}{ }^{1,2, \#}$, Xiao-yu FAN², Lei CAO ${ }^{1,2}$, Rui CHEN², Ya-jun GUO 1, 2, 3, *, Jian ZHAO ${ }^{2, *}$ \\ ${ }^{1}$ School of Medicine and School of Pharmacy, Shanghai Jiao Tong University, Shanghai 200025, China; ${ }^{2}$ International Joint Cancer \\ Institute, The Second Military Medical University, Shanghai 200433, China; ${ }^{3}$ PLA General Hospital Cancer Center, PLA Postgraduate \\ School of Medicine, Beijing 100863, China
}

Aim: Osteopontin (OPN), a multifunctional protein, has been reported to be protoxicant in acetaminophen hepatotoxicity. In this study, the mechanisms underlying the detrimental role of OPN in acetaminophen toxicity were explored.

Methods: Male C57BL/6 (wild-type, WT) and $\mathrm{OPN}^{-/-}$mice were administered with acetaminophen (500 mg/kg, ip). After the treatment, serum transaminase (ALT), as well as OPN expression, histology changes, oxidative stress and inflammation response in liver tissue were studied. Freshly isolated hepatocytes of WT and $\mathrm{OPN}^{-/-}$mice were prepared.

Results: Acetaminophen administration significantly increased OPN protein level in livers of WT mice. OPN expression was mainly localized in hepatic macrophages $6 \mathrm{~h}$ after the administration. In $O P N^{-1-}$ mice, acetaminophen-induced serum ALT release was reduced, but the centrilobular hepatic necrosis was increased. In OPN ${ }^{-/-}$mice, the expression of CYP2E1 and CYP1A2 in livers was significantly increased; GSH depletion and lipid peroxidation in livers were enhanced. On the other hand, $O P N^{-1-}$ mice exhibited less macrophage and neutrophil infiltration and reduced expression of proinflammatory cytokines TNF- $\alpha$ and IL-1 $\alpha$ in livers. An anti-OPN neutralizing antibody significantly reduced acetaminophen-induced serum ALT level and inflammatory infiltration in livers of WT mice. Conclusion: OPN plays a dual role in acetaminophen toxicity: OPN in hepatocytes inhibits acetaminophen metabolism, while OPN in macrophages enhances acetaminophen toxicity via recruitment of inflammatory cells and production of proinflammatory cytokines.

Keywords: acetaminophen; osteopontin; liver; hepatotoxicity; macrophage; hepatocyte; cytokine

Acta Pharmacologica Sinica (2012) 33: 1004-1012; doi: 10.1038/aps.2012.47; published online 25 Jun 2012

\begin{abstract}
Introduction
Acetaminophen (APAP), an effective analgesic and antipyretic drug, is safe at therapeutic doses. However, overdoses of APAP commonly cause drug-induced liver failure ${ }^{[1]}$. At therapeutic doses, about $90 \%$ of APAP is conjugated through sulfation or glucuronidation and then excreted into bile and urine, whereas $5 \%-10 \%$ of APAP is oxidized into $N$-acetylpbenzoquinone imine (NAPQI) by cytochrome P450s (CYPs). NAPQI is inactivated by conjugation with reduced glutathione (GSH) to form a 3-S-glutathionyl conjugate of APAP. At toxic doses, sulfation and glucuronidation pathways become saturated; hence, more NAPQI are formed with rapid depletion of hepatic GSH and then covalently bind to cellular proteins. APAP hepatotoxicity occurs after the depletion of GSH
\end{abstract}

\footnotetext{
\# The first two authors contributed equally to this work.

* To whom correspondence should be addressed.

E-mail zhaojian@smmu.edu.cn (Jian ZHAO); yjguo@smmu.edu.cn (Ya-jun GUO)

Received 2012-02-22 Accepted 2012-04-01
}

stores $^{[2]}$. In mice and hamsters, phase I biotransformation is mostly limited to the P450 enzymes, CYP2E1 and CYP1A2 $2^{[2]}$. CYP2E1 is a major CYP contributing to the metabolism of acetaminophen to NAPQI. CYP2E1 knockout mice was more resistant to APAP hepatotoxicity than WT mice ${ }^{[3,4]}$. CYP2E1 is the main CYP that bioactivates APAP at low doses ${ }^{[5]}$. At a higher dose, CYP1A2 was shown to contribute to the bioactivation and toxicity of APAP ${ }^{[2]}$. Mice deficient in CYP2E1 and CYP1A2 exhibited high resistance to APAP hepatotoxicity $^{[6]}$. CYP3A11 was also implicated in APAP hepatotoxicity ${ }^{[7]}$. Thus, genetic modulation of CYPs may influence the susceptibility to APAP toxicity.

Osteopontin (OPN), a highly modified integrin-binding extracellular matrix glycophosphoprotein, has been implicated in cell signaling that controls inflammation, tumor progression, and metastasis ${ }^{[8]}$. Recently, OPN was shown to act as a key stress protein in mechanic, oxidative and physical stress $^{[9-11]}$. OPN mediates diversely biological activities, such as cell survival, motility and proliferation, through interaction with certain integrins and CD44 variants with its arginine- 
glycine-aspartate (RGD) and non-RGD motifs. The activities of OPN are closely involved in macrophage-mediated proinflammatory responses. OPN induces interleukin (IL)-12 in macrophages and suppresses anti-inflammatory cytokine IL-10 production, which results in the promotion of $\mathrm{T}$ helper cell type 1 differentiation ${ }^{[12]}$. OPN was shown to increase the expression of tumor necrosis factor (TNF)- $\alpha$ but not IL-6 or IL-1 $\beta$ in mouse resident peritoneal macrophages ${ }^{[13]}$. OPN is expressed in activated Kupffer cells (KCs), hepatic macrophages and stellar cells in response to $\mathrm{CCl}_{4}$ treatment ${ }^{[14]}$. The activation of KCs directly or indirectly mediates hepatic toxicity and carcinogenesis through the release of multiple inflammatory cytokines, growth factors, and reactive oxygen species $^{[15]}$. OPN was reported to modulate the ability of reactive oxygen species (ROS) production and the synthesis of inflammatory cytokines in KCs following a Propionibacterium acnes challenge ${ }^{[16]}$. APAP hepatotoxicity is frequently associated with inflammatory infiltration, and the nature and extent of the inflammation determine the progression and the severity of the injury ${ }^{[17]}$. Thus, the modulation of OPN on macrophage functions may influence APAP toxicology. OPN has been reported to play a protoxicant role in APAP-induced liver injury ${ }^{[18]}$. As demonstrated by microarray data, higher OPN mRNA was identified in APAP-sensitive strain C57BL/6 mice as compared to the resistant SJL mice after APAP treatment. OPN knockout mice were more resistant to APAP-mediated liver injury ${ }^{[18]}$. Nevertheless, the cellular origin of OPN and the mechanisms underlying its role in APAP hepatotoxicity remains unknown.

Accumulating evidence demonstrates that hepatic damage may be the event that triggers an immune response and then contributes to APAP toxicity. Despite increasing oxidative stress, the hepatocytes did not die after GSH depletion. Massive death of hepatocytes occurred $6 \mathrm{~h}$ after APAP administration and $4 \mathrm{~h}$ after GSH depletion ${ }^{[19]}$. Macrophages were shown to aggravate hepatic injury through the production of proinflammatory mediators, such as TNF- $\alpha$, IL-1a, and nitric oxide $^{[20,21]}$. The cytotoxic and inflammatory mediators generated by activated inflammatory cells may aggravate cell damage and promote APAP toxicity ${ }^{[22]}$. In various liver inflammation models, OPN is a chemotactic factor for macrophages and neutrophils ${ }^{[23]}$. OPN deficiency caused reduced macrophage accumulation in many diseases, such as renal injury and colitis ${ }^{[24]}$. Moreover, OPN is a critical chemoattractant for neutrophils in liver inflammation models ${ }^{[23]}$. Depletion of neutrophils before APAP treatment was reported to provide protection against APAP-induced liver injury ${ }^{[25]}$. In this study, we explore the role of OPN in APAP metabolism and inflammation-mediated liver injury.

\section{Materials and methods \\ Mice}

C57BL/ 6 mice were purchased from the Shanghai Experimental Animal Center of Chinese Academic of Sciences (Shanghai, China). OPN $N^{-/-}$mice (B6.Cg-Spp1tm1blh/J, Cat № 004936) were obtained from the Jackson Laboratory (Genetics research,
Bar Harbor, Maine, USA). All animals in this study were kept and bred in the Animal Unit of Shanghai Second Military Medical University in environmentally controlled and specific pathogen-free conditions.

The animals received considerate human care. All animal experimental procedures and protocols were approved by and conducted in accordance with the guidelines of the Animal Experiment Committee of the Shanghai Second Military Medical University of China.

\section{Animal treatment}

Eight-week-old male C57BL/6 and $\mathrm{OPN}^{-/-}$mice were intraperitoneally administered APAP (500 mg/ $\mathrm{kg}$, dissolved in PBS) and sacrificed at the indicated time. The mouse $\mathrm{mAb} 23 \mathrm{C} 3$ against human OPN were generated in our laboratory. The mouse mAb $23 \mathrm{C} 3$ showed cross-reactivity with mouse OPN ${ }^{[26]}$. For anti-OPN antibody treatment, $200 \mu \mathrm{g}$ of anti-OPN Ab or mouse IgG (Sigma, St Louis, MO, USA) was intraperitoneally injected $2 \mathrm{~h}$ before APAP administration. Serum was collected for transaminase (ALT) assay and aspartate aminotransferase (AST) assay. The livers were rapidly removed and snap frozen in liquid nitrogen for RNA isolation, OPN quantification, and biochemical analysis or fixed in $10 \%$ neutral formalin buffer for histological assay.

\section{Quantification of OPN protein in liver tissues}

The livers were removed and snap frozen in liquid nitrogen. The frozen livers were homogenized in ice-cold cell lysis buffer (Cell Signaling, Danvers, MA, USA). After centrifugation at $20000 \times g$ for $30 \mathrm{~min}$ at $4{ }^{\circ} \mathrm{C}$, supernatant was pooled for OPN qualification using mouse OPN ELISA kit (R\&D Systems, Minneapolis, MN, USA). The total protein was quantified using the BCA kit (Pierce, Rockford, IL, USA).

\section{RNA isolation and quantitative real-time PCR}

Total liver RNA was isolated using the Nucleospin RNA (Macherey-Nagel, Germany). The first strand synthesis was performed with random primers and reverse transcription with Quant Reverse Transcriptase (Tiangen Biotech, China). The quantitative real-time PCR was performed using a SYBR Green reagent in a Light Cycler (Roche, Germany). The reactions were performed twice in triplicate, and actin values were used to normalize gene expression. The primer sequences are presented in the Supplementary Data (Table 1).

\section{Biochemistry analysis}

Serum ALT and AST levels were measured with a colorimetric endpoint method utilizing diagnostic reagent kits (Pointe Scientific Inc, Canton, MI, USA) according to the manufacturer's protocol using a Roche Cobas Mira Classic Chemistry Analyzer (Roche Diagnostic systems, Inc, Branchburg, NJ, USA). ALT and AST levels were expressed as units per liter of serum. For GSH and myeloperoxidase (MPO) assay, liver tissue was weighed and homogenized in cold phosphate buffer (20 $\mathrm{mmol} / \mathrm{L}, \mathrm{pH}$ 7.2). For GSH analysis, homogenized liver was centrifuged at $10000 \times g$ for $10 \mathrm{~min}$ at $4^{\circ} \mathrm{C}$. Supernatant was 
Table 1. Primers for real-time PCR.

\begin{tabular}{|c|c|c|}
\hline Gene & Primer & Sequence \\
\hline \multirow{2}{*}{ mACTIN } & Forward & 5'-TGTTACCAACTGGGACGACA-3' \\
\hline & Reverse & 5'-CTGGGTCATCTTTTCACGGT-3' \\
\hline \multirow[t]{2}{*}{ mCYP2E1 } & Forward & 5'-ССТTTCССААТTССТTTCTTTG-3' \\
\hline & Reverse & 5'-TCTTGTGGTTCAGTAGCACCTCC-3' \\
\hline \multirow[t]{2}{*}{$m C Y P 1 A 2$} & Forward & 5'-AGTACATCTCCTTAGCCCCAG-3' \\
\hline & Reverse & 5'-GGTCCGGGTGGATTCTTCAG-3' \\
\hline \multirow[t]{2}{*}{ mCYPЗА11 } & Forward & 5'-ATGGAGATCACAGCCCAGTC-3' \\
\hline & Reverse & 5'-ATGCAGGGTGAAGGAAAGTG-3' \\
\hline \multirow[t]{2}{*}{ mIL6 } & Forward & 5'-GCTACCAAACTGGATATAATCAGGA-3' \\
\hline & Reverse & 5'-CCAGGTAGCTATGGTACTCCAGAA-3' \\
\hline \multirow[t]{2}{*}{$m T N F-\alpha$} & Forward & 5'-ACTCAAATGGGCTTTCCGAAT-3' \\
\hline & Reverse & 5'-CACAGGGAAGAATCTGGAAAGG-3' \\
\hline \multirow[t]{2}{*}{$m I L-1 a$} & Forward & 5'-TGCATGGCATTCTTAGGAGG-3' \\
\hline & Reverse & 5'-TCAACTGGCATTTTGAAGCC-3' \\
\hline
\end{tabular}

used for the quantification of GSH level using a commercial kit (Nanjing Jiancheng Biotech, China). Total protein in the supernatant was quantified using a BCA kit (Pierce). GSH levels were expressed as microgram per gram of protein. MPO activity in liver homogenate was measured using commercial kits (Nanjing Jiancheng Biotech, China) and expressed as units per gram of liver. For malondialdehyde (MDA) analysis, liver tissue was weighed and homogenized in Tris- $\mathrm{HCl}$ buffer $(20$ $\mathrm{mmol} / \mathrm{L}, \mathrm{pH}$ 7.4). MDA in homogenate was measured using commercial kits (Nanjing Jiancheng Biotech, China). MDA content was expressed as micromole per gram of liver.

\section{Histochemical analysis}

Liver tissue was removed from mice with different treatment. Liver samples were fixed in $10 \%$ neutral formalin buffer and embedded in paraffin wax, and the sections were stained with H\&E. The tissue sections were examined under a light microscope and photographed using a Nikon camera fitted to the microscope. Images were acquired as mentioned above, and the quantitative data were obtained using a computerized image analysis system (KS 300, Carl Zeiss Vision). The analysis was performed on an average of 25 fields per section using $\times 10$ objective. The necrosis was expressed as a percentage of necrotic areas per field area.

The expression of OPN protein in mouse livers was detected by immunohistochemistry (IHC) with mouse anti-OPN mAb $23 \mathrm{C} 3$ as the primary antibody and rabbit anti-mouse IgG as the secondary antibody. The expression of F4/80 in mouse livers was detected by a rat anti-mouse F4/80 antibody (Cell Signaling, Danvers, MA, USA) and Cy3-labeled anti-rat IgG (Beyotime, China) as the primary and secondary antibody, respectively. Nuclei were visualized by DAPI staining. F4/80 staining was observed by fluorescent microscopy and photographed. The number of F4/80 positive cells per field was determined in the centrilobular areas by morphometric analysis using the SPOT advanced software package (Diagnostic Instruments, Inc, Sterling Heights, MI, USA). The analysis was performed on an average of 10 fields per section using the $\times 40$ objective.

\section{Isolation of liver cells and cell culture}

Hepatocytes were isolated using a standard collagenase procedure as described ${ }^{[27]}$. The hepatocyte viability, as determined by trypan blue exclusion, was generally $>95 \%$, and the cell purity was $>95 \%$. Cells were plated in collagen I-coated 12 -well plates $\left(6 \times 10^{5}\right.$ cells per well) (BD BioCoat ${ }^{\mathrm{TM}}$, BD Biosciences, San Jose, CA, USA) in HepatoZYME-SFM medium (Gibco, Grand Island, NY, USA) containing $100 \mathrm{U} / \mathrm{mL}$ penicillin/streptomycin and cultured at $37^{\circ} \mathrm{C}$ in $5 \% \mathrm{CO}_{2}$. After 3-h attachment, the cultures were washed with PBS and then plain culture medium (controls) or media containing $100 \mu \mathrm{mol} / \mathrm{L}$ $\mathrm{H}_{2} \mathrm{O}_{2}$ were added for $6 \mathrm{~h}$ or $12 \mathrm{~h}$. Cell viability was measured with MTS-based assay (Promega, Southampton, UK) according to the manufacturer's instructions.

\section{Statistical analysis}

Data are expressed as mean \pm SEM. Differences were analyzed by Student's $t$ test, and $P$ values $<0.05$ were considered significant.

\section{Results}

\section{OPN expression was increased after APAP administration}

Wild-type (WT) mice were ip injected with a toxic dose of APAP and OPN protein in livers was assayed at different time points (Figure 1A). OPN protein increased $2 \mathrm{~h}$ later and doubled $6 \mathrm{~h}$ after APAP administration. The OPN level in livers remained high $12 \mathrm{~h}$ after APAP treatment.

We detected the distribution of OPN in liver using IHC staining. The normal liver did not show OPN staining (Figure 1B). Six hours after APAP exposure, strong positive staining
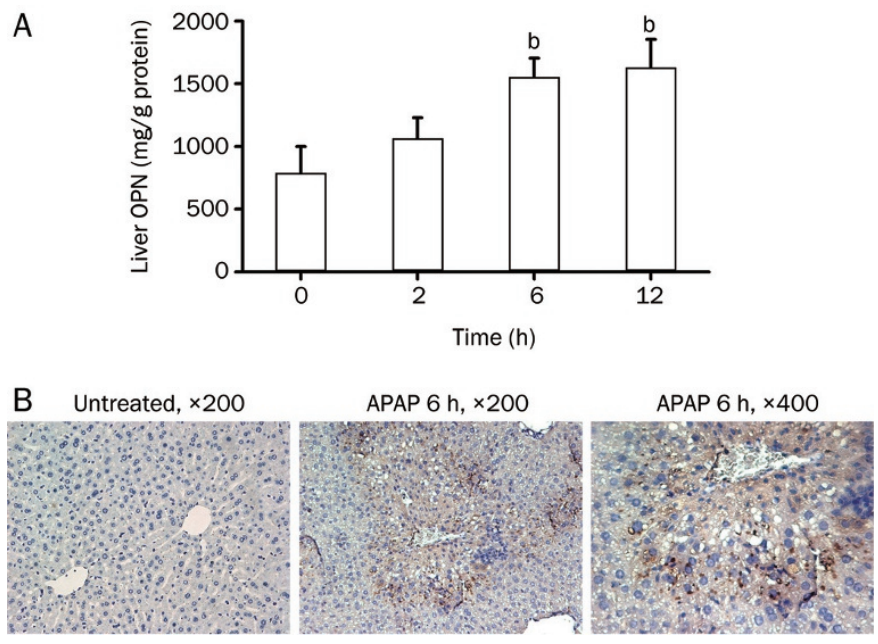

Figure 1. OPN expression in livers of wild-type (WT) male mice after APAP administration. (A) WT male mice were ip administered with 500 $\mathrm{mg} / \mathrm{kg}$ APAP and sacrificed at different time. Livers were removed for the measurement of OPN protein level. $n=5$ for each time point; (B) Immunohistochemical staining for OPN in the livers of WT mice $6 \mathrm{~h}$ after APAP administration. Data are mean \pm SEM. ${ }^{b} P<0.05$ vs $0 \mathrm{~h}$. 
was observed in hepatic macrophages adjacent to necrotic hepatocytes in Zone III (Figure 1B). OPN expression was mainly localized in hepatic macrophages. We also observed a slight increase of OPN expression in necrotic hepatocytes.

These results indicate that OPN expression was increased after APAP administration and this increase is mainly localized in hepatic macrophages.

OPN deficiency mice exhibited reduced serum ALT but more necrosis after APAP treatment

To determine the involvement of OPN in APAP hepatotoxicity, WT and $O P N^{-1-}$ male mice were injected with APAP and serum ALT was assayed at different time points. As previously reported $^{[18]}$, OPN knockout mice showed significantly lower ALT levels than WT mice (Figure 2A). The maximum ALT appeared $6 \mathrm{~h}$ after APAP treatment in $O P N^{-/-}$mice and rapidly recovered to the basal level. In comparison, serum ALT in WT mice was twice that of the $O P N^{-/-}$mice at $6 \mathrm{~h}$ and reached the maximum $24 \mathrm{~h}$ after APAP treatment. Both ALT and AST levels in WT and $O P N^{-/-}$mice exhibited similar trend (Figure 2B).

We further examined the histopathology of the livers of APAP-treated mice. APAP causes severe hepatic damage with massive necrotic hepatocytes in the central lobular zones (Figure $2 \mathrm{C}$ ). The necrosis area of the livers from $O P N^{-1-}$ mice was significantly greater than that of WT mice (Figure 2D), which was inconsistent with the results of serum ALT. The discrepancy between the ALT results and the histological grades was also observed in other studies ${ }^{[28]}$.

The data indicated that OPN enhanced the release of serum ALT level despite the inhibition of necrosis.
OPN deficiency enhanced APAP metabolism and oxidative stress APAP-induced hepatic necrosis was closely related to the oxidative stress caused by APAP metabolism ${ }^{[19]}$. To examine whether OPN deficiency enhanced the formation of NAPQI, the key CYP enzymes involved in APAP metabolism were analyzed. CYP2E1 is an important CYP in APAP toxicity ${ }^{[3]}$. APAP treatment caused the reduction of CYP2E1 mRNA expression, whereas $O P N^{-/-}$mice showed significantly higher CYP2E1 expression than WT mice, $2 \mathrm{~h}$ and $6 \mathrm{~h}$ after treatment (Figure 3A). At toxic doses, CYP1A2 and CYP3A may be the predominant CYPs in APAP oxidation. When untreated, $O P N^{-/-}$mice exhibited higher CYP1A2 expression than WT mice (Figure 3B). APAP treatment remarkably increased the expression of CYP1A2. The expression of CYP1A2 was significantly higher in APAP-exposed $O P N^{-/-}$mice than WT mice. The basal level of CYP3A11 expression in $\mathrm{OPN}^{-/-}$mice was lower than that of WT mice. However, no significant difference was observed in CYP3A11 expression between the groups after APAP treatment (Figure 3C).

Higher level of CYPs is related to increased formation of NAPQI, which results in the depletion of reduced GSH. The hepatic GSH levels were measured at 0,2 , and $6 \mathrm{~h}$ after APAP treatment. OPN deficiency did not cause a significant change on the basal GSH level (Figure 3D). APAP treatment resulted in GSH depletion in both mice. Interestingly, a significantly higher GSH level was observed in WT mice at $2 \mathrm{~h}$ and $6 \mathrm{~h}$ after APAP treatment compared to $O P N^{-/-}$mice. At $6 \mathrm{~h}$, the hepatic $\mathrm{GSH}$ level in $O P N^{-1-}$ mice was fully restored to the pretreatment level while the GSH level in WT mice was slightly upregulated by about $10 \%$ above the pretreatment level. Oxidative stress following GSH depletion was often accompanied
A

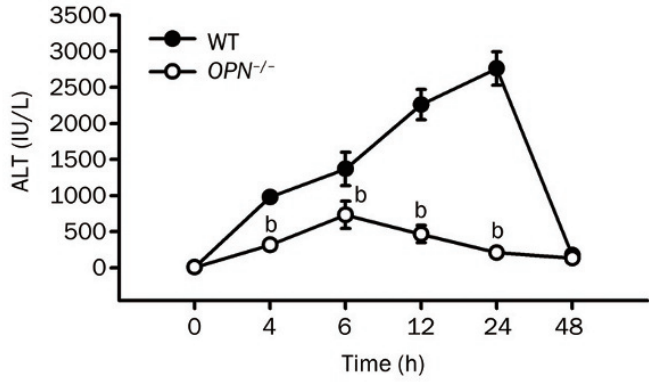

C

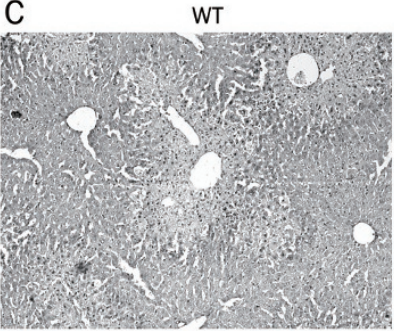

$\mathrm{OPN}^{-1}$

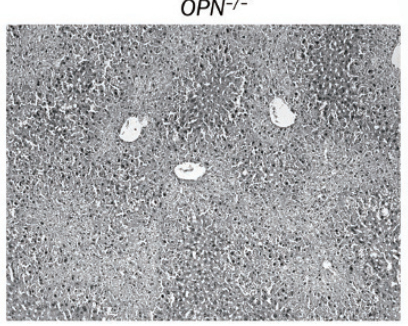

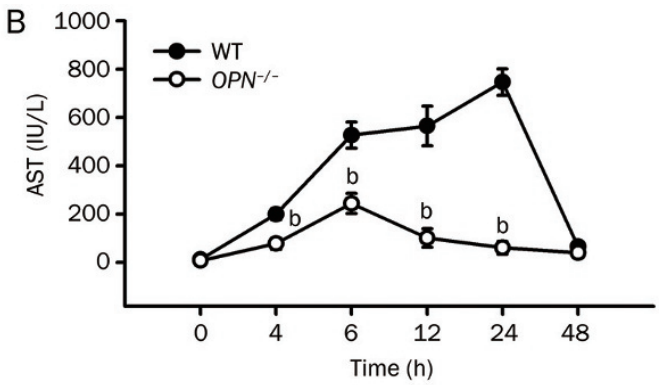

D

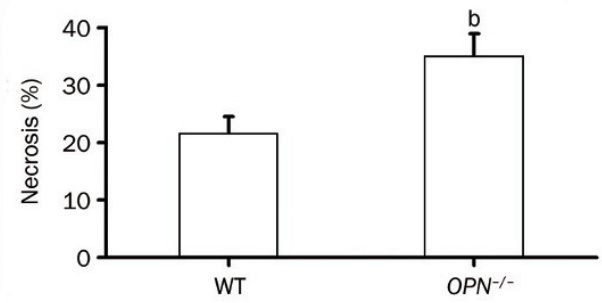

Figure 2. Serum ALT and AST level and histologic necrosis in APAP-treated WT and $O P N^{-1-}$ mice. (A and B) WT male and OPN ${ }^{-1-}$ mice were given APAP (500 $\mathrm{mg} / \mathrm{kg}$ ), and ALT level (A) and AST (B) were measured at indicated time points. ( $n=6$ mice per time point). (C and D) Representative hematoxylin/eosin staining of liver section (C) (200×magnification) and average necrosis (D) $6 \mathrm{~h}$ after APAP treatment. Data are mean \pm SEM. ${ }^{\mathrm{b}} \mathrm{P}<0.05 \mathrm{vs}$ WT. 

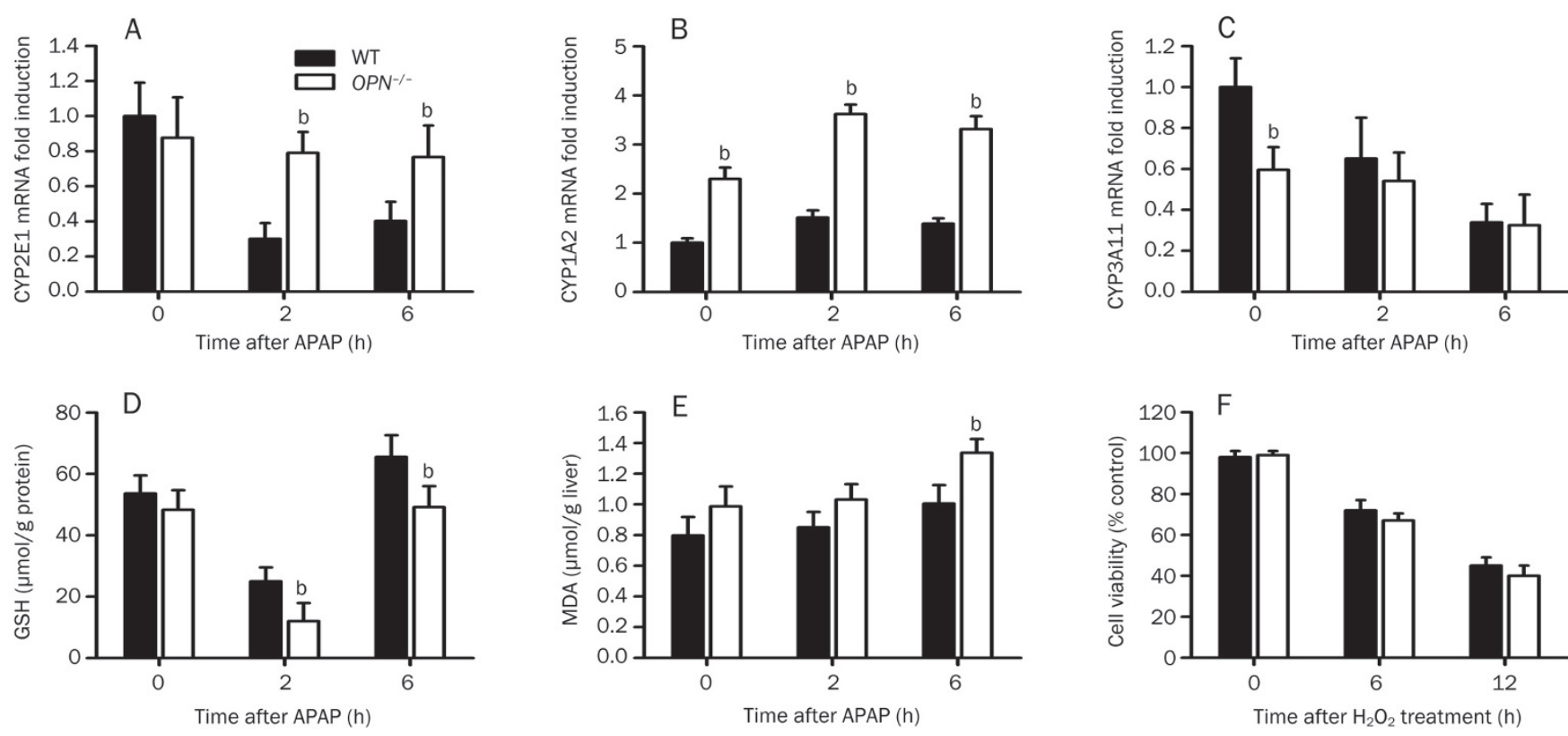

Figure 3. Effects of OPN deficiency on the expression of CYPs genes and redox homeostasis after APAP administration. WT or OPN ${ }^{-/-}$mice ( $\left.n=4\right)$ were treated with APAP and their livers were removed at indicated time. (A-C) The expression of CYP2E1 (A), CYP1A2 (B), and CYP3A11 (C) in livers after APAP administration were detected by real-time PCR. Actin values were used to normalize gene expression. (D and E) liver lysates were analyzed for GSH content (D) and MDA content (E). $n=4$ for each time point. (F) Viability of WT and $\mathrm{OPN}^{-/-}$hepatocytes after $\mathrm{H}_{2} \mathrm{O}_{2}$ treatment. Freshly isolated WT and $\mathrm{OPN}^{-/-}$hepatocytes were inoculated with or without $100 \mu \mathrm{mol} / \mathrm{L} \mathrm{H}_{2} \mathrm{O}_{2}$ and cell viability was measured with MTS assay at different time points. Data are mean \pm SEM. ${ }^{\mathrm{b}} P<0.05$, Student's $t$-test.

by lipid peroxidation (LPO). The LPO, measured by MDA level (Figure 3E), was not significantly increased in APAPtreated WT mice, indicating relatively low lipid peroxidation. The MDA level of $O P N^{-/-}$mice was significantly higher than that of WT mice $6 \mathrm{~h}$ after APAP exposure.

We further examine whether OPN deficiency enhances the susceptibility of hepatocytes to oxidative stress. Using isolated hepatocytes, we found that $O P N^{-/-}$hepatocytes did not exhibit significantly higher necrosis than WT hepatocytes under $\mathrm{H}_{2} \mathrm{O}_{2}$ treatment (Figure 3F). Thus, OPN deficiency did not enhance the sensitivity of hepatocytes to oxidative stress.

These results show that OPN deficiency accelerated the formation of reactive metabolites and enhanced oxidative stress, which partly resulted in enhanced hepatic necrosis.

OPN promotes APAP toxicity through the enhancement of inflammatory accumulation and the production of proinflammatory mediators

Although the oxidative stress caused by APAP metabolism leads to direct hepatic cellular dysfunction and death, the release of a variety of inflammatory mediators may influence individual susceptibility. OPN is chemotactic for macrophages in liver inflammation ${ }^{[23]}$. Using F4/80 IHC, greater macrophage accumulation was observed in WT mice than in $\mathrm{OPN}^{-/-}$mice $6 \mathrm{~h}$ after APAP administration (Figure 4). Hepatic macrophage was implicated in APAP toxicity as a result of the production of a variety of proinflammatory cytokines $^{[29]}$. We examined the expression of TNF-a, IL-1a, and
IL-6 expression in APAP-treated mice. Similar to previous reports ${ }^{[30]}$, APAP administration greatly enhanced TNF- $\alpha$ and IL-1a expression. OPN deficiency resulted in lower TNF- $a$ and IL-1a expression in $O P N^{-/-}$mice compared with WT mice after APAP treatment (Figure 5A). This lower production of proinflammatory cytokines coincided with reduced macrophage infiltration. IL-6 expression did not change significantly after APAP administration in both WT mice and $O P N^{-/-}$ mice (Figure 5C).

OPN plays a role in the recruitment of neutrophils in liver inflammation ${ }^{[23]}$. We examined the neutrophil infiltration in the livers of the treated mice. The neutrophil accumulation, evidenced by MPO level (Figure 5D), was increased in WT mice after APAP and correlated with OPN expression. In comparison, the MPO level did not significantly increase after APAP administration in $O P N^{-/-}$mice. This result shows that OPN is critical for APAP-induced neutrophil recruitment.

These results indicate that OPN promotes APAP hepatotoxicity through the enhancement of inflammatory infiltration and proinflammatory cytokine expression.

Anti-OPN antibody reduced hepatic damage through the inhibition of inflammatory infiltration

To corroborate the role of OPN in APAP toxicity, we examined the therapeutic activity of anti-OPN antibody. The antiOPN neutralizing antibody, 23C3, was shown to reduce hepatic damage through inhibition of NK and NKT cell infiltration in mural ConA-induced hepatitis model ${ }^{[31]}$. WT mice 

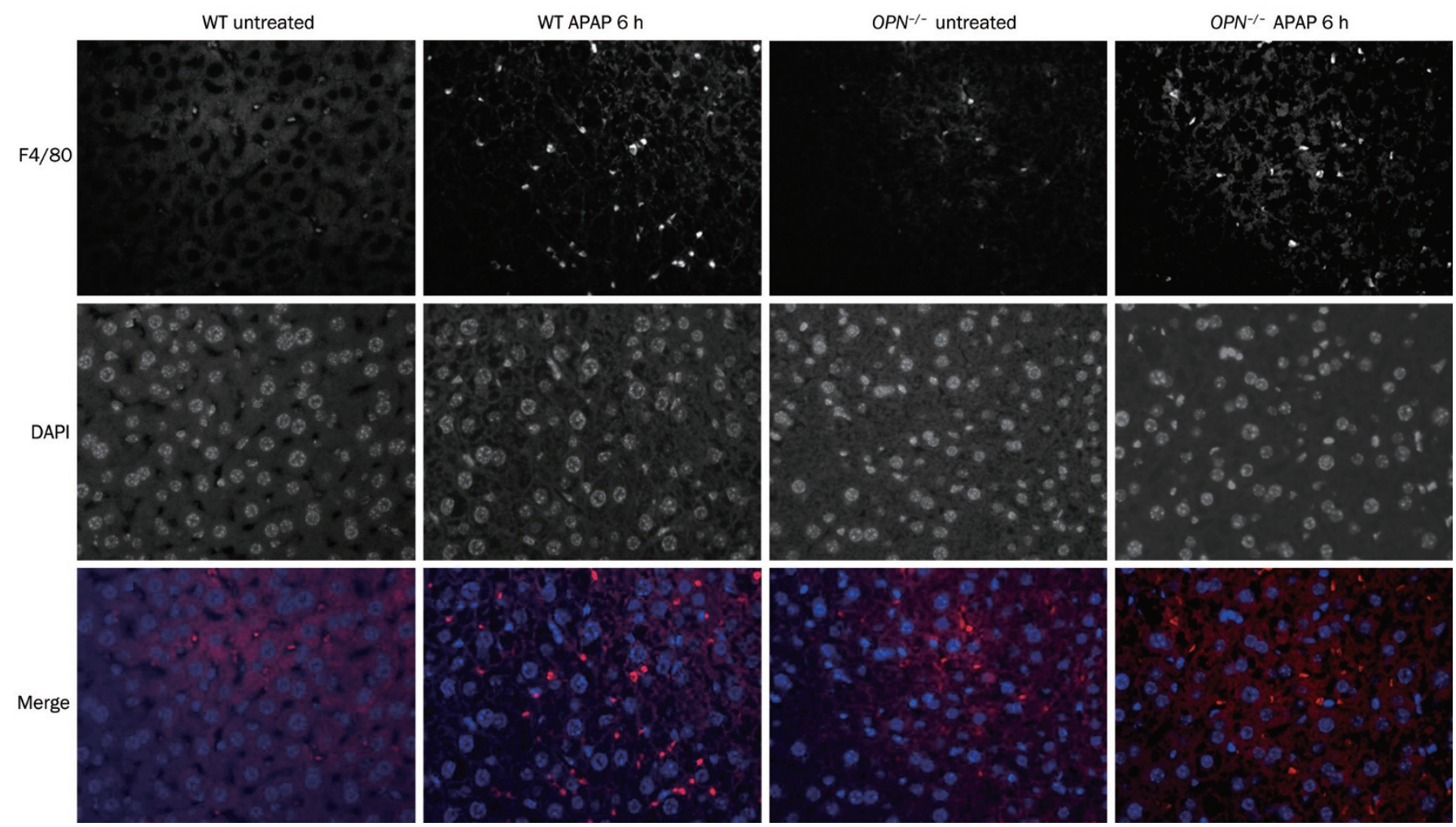

Figure 4. F4/80 staining in livers of APAP-treated mice. WT and OPN ${ }^{-/-}$mice were administered with APAP. Six hours later, the expressions of F4/80 in mouse livers were detected by rat anti-mouse F4/80 antibody and Cy3-labeled anti-rat IgG as primary and secondary antibody, respectively. Nuclei were visualized by DAPI staining $(\times 400)$.
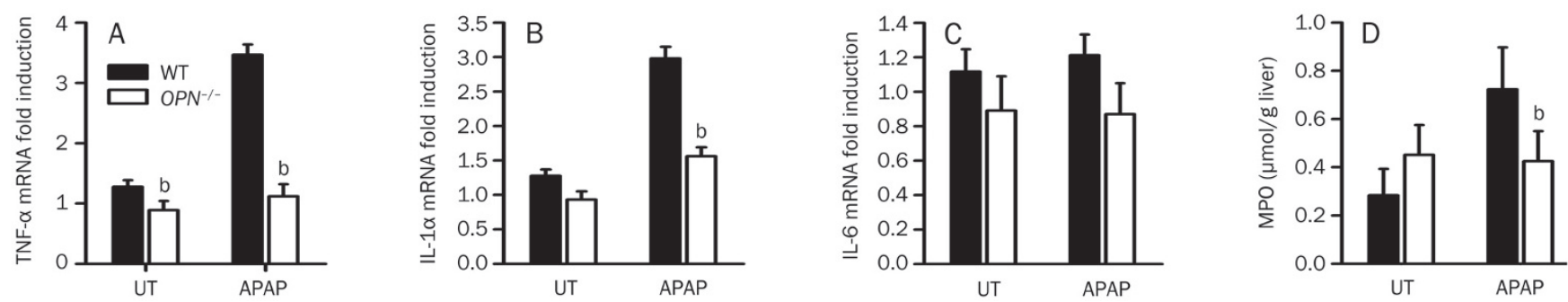

Figure 5. Effects of OPN deficiency on proinflammatory cytokines and neutrophil infiltration. WT or OPN ${ }^{-/-}$mice $(n=4)$ were treated with APAP and their livers were removed $6 \mathrm{~h}$ later. $(A-C)$ The expression of TNF- $\alpha(A), I L-1 \alpha(B)$, and IL- $6(C)$ in livers were detected by real-time PCR. Actin values were used to normalize gene expression. (D) Liver lysates were analyzed for MPO level $6 \mathrm{~h}$ after APAP. $n=4$ for each time point. Data are mean \pm SEM. ${ }^{\mathrm{b}} P<0.05$, Student's t-test.

were pretreated with $23 \mathrm{C} 3$ and then challenged with toxic doses of APAP. Pretreatment with $23 \mathrm{C} 3$ in WT mice significantly reduced ALT level (Table 2). Similarly, the accumulation of F4/80 positive cells and the MPO level were markedly reduced as a result of $23 \mathrm{C} 3$ pretreatment (Table 2). Moreover, 23C3 also reduces APAP-induced hepatocellular necrosis, although not significantly. The anti-OPN neutralizing antibody effectively prevented APAP-induced hepatotoxicity through the inhibition of inflammatory infiltration.

\section{Discussion}

The pathogenesis of APAP-induced hepatotoxicity consists of direct cellular damage as well as the innate immune system. Our study demonstrates that OPN plays a dual role in APAP hepatotoxicity.

The mechanism of APAP detoxification and the sensitivity of hepatocytes may have a direct effect on hepatocyte death. The level of hepatic phase I oxidative enzymes is correlated with the sensitivity of APAP toxicity. CYP2E1 and CYP1A2 are the two major CYPs in APAP metabolism in mice ${ }^{[6]}$. OPN deficiency enhanced the expression of both CYP2E1 and CYP1A2, which may accelerate the production of the reactive metabolite. Increased oxidative stress caused higher GSH depletion and lipid peroxidation. Because the metabolism 
Table 2. Pretreatment with anti-OPN antibody $23 \mathrm{C} 3$ reduced ALT, histological necrosis, macrophage and neutrophil infiltration in WT mice $6 \mathrm{~h}$ after APAP administration. $n=6$ for each group. ${ }^{b} P<0.05$ compared to lgG-treated mice. ${ }^{*} N D$, Not done.

\begin{tabular}{|c|c|c|c|c|c|c|c|c|}
\hline \multirow{2}{*}{ Treatment } & \multicolumn{2}{|c|}{ ALT (IU/L) } & \multicolumn{2}{|c|}{ Necrosis \% } & \multicolumn{2}{|c|}{ F4/80 positive cells per field } & \multicolumn{2}{|c|}{ MPO ( $\mu \mathrm{mol} / g$ tissue) } \\
\hline & $\lg G$ & $23 \mathrm{C3}$ & IgG & $23 \mathrm{C3}$ & $\lg G$ & $23 \mathrm{C3}$ & $\lg G$ & $23 \mathrm{C3}$ \\
\hline Untreated & $6 \pm 1$ & $5.4 \pm 1.2$ & ND & ND & $N D^{*}$ & ND & $0.30 \pm 0.09$ & $0.32 \pm 0.11$ \\
\hline APAP & $1440 \pm 80$ & $870 \pm 120^{b}$ & $23 \pm 4$ & $18 \pm 4$ & $37 \pm 5$ & $22 \pm 3^{b}$ & $0.75 \pm 0.18$ & $0.35 \pm 0.15^{b}$ \\
\hline
\end{tabular}

of APAP occurs mainly in hepatocytes, OPN deficiency may interfere with the bioactivation of APAP. OPN can be synthesized in hepatocytes in low levels ${ }^{[32]}$ and can be upregulated under pathologic conditions ${ }^{[33]}$. Recent studies have revealed that an intracellular form of OPN (iOPN), except the secreted isoform of OPN (sOPN), regulates cell motility, cell division and cytokine expression ${ }^{[34]}$. Nevertheless, the mechanism by which hepatocyte-derived iOPN or sOPN modulates CYP gene expression requires further investigation. Another possible reason for enhanced necrosis observed in $\mathrm{OPN}^{-/-}$mice is the prosurvival activity of OPN. Necrosis is the main mode of hepatocyte death after APAP overdose $\mathrm{e}^{[35]}$. OPN was reported to inhibit cardiac fibroblast necrosis in response to hydrogen peroxide $\left(\mathrm{H}_{2} \mathrm{O}_{2}\right)$ in caspase-3-independent pathway. Under $\mathrm{H}_{2} \mathrm{O}_{2}$ treatment, OPN ${ }^{-/-}$hepatocytes did not exhibit higher necrosis than WT hepatocytes (Figure 3F). This inconsistency may be due to the relatively low expression of OPN in hepatocytes. Thus, increased hepatocyte necrosis in $O P N^{-/-}$mice may be explained by the enhanced oxidative stress caused by APAP metabolism.

Serum ALT is routinely measured as part of the diagnostic evaluation of hepatocellular injury. Although they may be identified as necrotic, not all necrotic hepatocytes die with the release of ALT. Thus, it is not surprising to observe a discrepancy between the necrosis and the ALT results in reports ${ }^{[28,36,37]}$. On the basis of ALT results, we conclude that OPN-mediated inflammatory response plays an important role in the progression of APAP hepatotoxicity. After GSH depletion, stressed hepatocytes release damage signals, such as $\mathrm{HMGB1}^{[30]}$ and IL-1a ${ }^{[38]}$, which activate $\mathrm{KCs}$ and recruit inflammatory cells. KCs activate the expression of TNF- $\alpha$, IL-6, and IL- $1 \beta$ as early as $1 \mathrm{~h}$ after APAP challenge ${ }^{[30]}$. The expression of OPN was reported to be upregulated in APAP toxicology, whereas the cells expressing OPN within the liver is unclear ${ }^{[18]}$. We found that activated KCs around necrotic hepatocytes were also the main source of OPN in livers after overdose APAP exposure. As a critical chemotactic factor for macrophages, the upregulation of OPN at sites of injury enhances macrophage infiltration. This macrophage accumulation can be effectively blocked by the pretreatment with anti-OPN antibody. Thus, OPN deficiency markedly reduced APAP-induced macrophage infiltration. The production of cytotoxic mediators by macrophages was one of the mechanisms underlying immune-mediated liver injury ${ }^{[17]}$. OPN deficiency in APAP-exposed mice resulted in the dysfunction of cytokine production. In comparison to WT mice, OPNdeficient mice exhibited markedly reduced production of proinflammatory cytokine TNF- $\alpha$ and IL-1a after APAP challenge. TNF-a has been linked to increased oxidative stress, such as ROS and nitric oxide ${ }^{[39,40]}$, and it is known to recruit and activate other inflammatory cells ${ }^{[41]}$. IL-1ra knockout mice exhibited a reduction in APAP toxicity with less liver injury and reduced leukocytes infiltration ${ }^{[42]}$. APAP hepatotoxicity was partially reduced by treatment with either anti-TNF-a or anti-IL-1a in acetaminophen-intoxicated mice ${ }^{[43]}$. Nonetheless, the role of OPN in the modulation of macrophage function requires further investigation. Moreover, both TNF- $a$ and IL-6 also contributed to the restoration of liver function by promoting liver regeneration ${ }^{[40,44]}$. The enhanced liver repair does not contribute to the reduced hepatic damage in $\mathrm{OPN}^{-/-}$mice, which showed reduced expression of TNF- $\alpha$ and IL-6 when compared to WT mice. Therefore, OPN may promote APAP toxicity through the enhancement of macrophage accumulation and the production of proinflammatory cytokines.

The primary role of neutrophil influx in APAP toxicity is postulated to serve to remove damaged cells and cellular debris $^{[45]}$. Although the detrimental role of neutrophils in APAP hepatotoxicity in animal models is still controversial, the recruited neutrophils may likely aggravate the injury by attacking and killing some of the stressed hepatocytes. Deletion of neutrophil using the anti-Gr-1 antibody was shown to significantly attenuate APAP toxicity in mice ${ }^{[25]}$. OPN is important for the migration of neutrophils in vitro and in $v i v 0^{[46]}$. Thus, neutrophil infiltration was not increased by APAP administration in $O P N^{-/-}$mice compared to WT mice. The inflammation and cytotoxic cytokines caused by elevated OPN production further aggravated the death of stressed hepatocytes. Consequently, serum ALT level in $O P N^{-1-}$ mice rapidly recovered to the basal level as a result of decreased inflammation, while increased inflammation in WT mice caused higher levels of serum ALT.

The dual role of OPN may be explained by its role in different liver cells during the process of APAP-induced liver injury. In the early stage, iOPN deficiency in hepatocytes caused increased CYP expression and enhanced APAP metabolisminduced oxidative stress. After hepatocyte damage, the elevation of sOPN in macrophages resulted in massive inflammatory infiltration and the release of proinflammatory cytokines. Pretreatment with anti-OPN antibody effectively inhibited APAP-induced inflammation infiltration with the reduction 
of serum ALT level through neutralizing sOPN. These results indicate that sOPN plays a protoxicant role in inflammationinduced liver injury, while iOPN in hepatocytes may play a hepatoprotective role. Thus, anti-OPN treatment may represent a promising therapy for APAP-induced liver diseases through the inhibition of inflammation-induced liver injury. OPN is a heterogeneous protein found with different degrees of phosphorylation in different cell types ${ }^{[47]}$. The function of OPN can be affected by its cell-specific post-translational modification, enzyme cleavage or different isoforms ${ }^{[34,48,49]}$. Hepatocyte-derived OPN may function differently from that produced by inflammatory cells. Hence, mice lacking OPN only in hepatocytes or in macrophages should be developed to examine the exact mechanism of OPN in APAP toxicity.

In conclusion, we find that OPN plays a dual role in APAP toxicity. At the APAP metabolism stage, iOPN in hepatocytes plays a protective role through the inhibition of APAP metabolism; however, at the inflammation stage, subsequent to metabolism, sOPN promotes inflammation-induced hepatic damage through the recruitment of macrophages and neutrophils. The anti-OPN antibody therapy may provide an effective treatment for APAP-induced liver diseases through inhibition of inflammation. Our findings may facilitate a better understanding of the mechanism underlying APAP toxicity and provide new avenues for the prevention and treatment of drug-induced liver injury.

\section{Acknowledgements}

This work is supported in part by grants from Ministry of Science and Technology of China (2010CB945600, 2011CB966200), National Natural Science Foundation of China, the Special Project for Infection Disease (2008ZX10002-019), New Drug Development and Program of Shanghai Subject Chief Scientists (10XD1405400).

\section{Author contribution}

Chun-yan HE (performing the major experiments and writing manuscript), Bei-bei LIANG (performing animal experiments), Xiao-yu FAN (RNA extraction and RT PCR), Lei CAO (expression of recombinant OPN and OPN antibody), Rui CHEN (Western blotting and IHC analysis), Jian ZHAO (analysis of data and manuscript writing), and Ya-jun GUO (study design, data analysis and writing the manuscript).

\section{Abbreviations}

ALT, transaminase; APAP, acetaminophen; AST, aspartate aminotransferase; CYPs, cytochrome P450s; GSH, glutathione; IHC, immunohistochemical; KC, Kupffer cell; LPO, lipid peroxidation; MDA, malondialdehyde; MPO, myeloperoxidase; NAPQI, $N$-acetyl-pbenzoquinone imine; OPN, osteopontin; RGD, arginine-glycine-aspartate.

\section{References}

1 Larson AM, Polson J, Fontana RJ, Davern TJ, Lalani E, Hynan LS, et al. Acetaminophen-induced acute liver failure: results of a United States multicenter, prospective study. Hepatology 2005; 42: 1364-72.
2 Bessems JG, Vermeulen NP. Paracetamol (acetaminophen)-induced toxicity: Molecular and biochemical mechanisms, analogues and protective approaches. Crit Rev Toxicol 2001; 31: 55-138.

3 Cheung C, Yu AM, Ward JM, Krausz KW, Akiyama TE, Feigenbaum L, et al. The cyp2e1-humanized transgenic mouse: role of cyp2e1 in acetaminophen hepatotoxicity. Drug Metab Dispos 2005; 33: 44957.

4 Chen C, Krausz KW, Idle JR, Gonzalez FJ. Identification of novel toxicity-associated metabolites by metabolomics and mass isotopomer analysis of acetaminophen metabolism in wild-type and Cyp2e1-null mice. J Biol Chem 2008; 283: 4543-59.

5 Lee SS, Buters JT, Pineau T, Fernandez-Salguero P, Gonzalez FJ. Role of CYP2E1 in the hepatotoxicity of acetaminophen. J Biol Chem 1996; 271: $12063-7$.

6 Zaher H, Buters JT, Ward JM, Bruno MK, Lucas AM, Stern ST, et al. Protection against acetaminophen toxicity in CYP1A2 and CYP2E1 double-null mice. Toxicol Appl Pharmacol 1998; 152: 193-9.

7 Guo GL, Moffit JS, Nicol CJ, Ward JM, Aleksunes LA, Slitt AL, et al. Enhanced acetaminophen toxicity by activation of the pregnane $X$ receptor. Toxicol Sci 2004; 82: 374-80.

8 Rangaswami H, Bulbule A, Kundu GC. Osteopontin: role in cell signaling and cancer progression. Trends Cell Biol 2006; 16: 79-87.

9 Ishijima M, Tsuji K, Rittling SR, Yamashita T, Kurosawa H, Denhardt DT, et al. Osteopontin is required for mechanical stress-dependent signals to bone marrow cells. J Endocrinol 2007; 193: 235-43.

10 Baliga SS, Merrill GF, Shinohara ML, Denhardt DT. Osteopontin expression during early cerebral ischemia-reperfusion in rats: enhanced expression in the right cortex is suppressed by acetaminophen. PLoS One 2011; 6: e14568.

11 Wang KX, Denhardt DT. Osteopontin: role in immune regulation and stress responses. Cytokine Growth Factor Rev 2008; 19: 333-45.

12 Ashkar S, Weber GF, Panoutsakopoulou V, Sanchirico ME, Jansson M, Zawaideh S, et al. Eta-1 (osteopontin): an early component of type-1 (cell-mediated) immunity. Science 2000; 287: 860-4.

13 Weber GF, Zawaideh S, Hikita S, Kumar VA, Cantor H, Ashkar S. Phosphorylation-dependent interaction of osteopontin with its receptors regulates macrophage migration and activation. J Leukoc Biol 2002; 72: 752-61.

14 Kawashima R, Mochida S, Matsui A, YouLuTuZ Y, Ishikawa K, Toshima $\mathrm{K}$, et al. Expression of osteopontin in Kupffer cells and hepatic macrophages and Stellate cells in rat liver after carbon tetrachloride intoxication: a possible factor for macrophage migration into hepatic necrotic areas. Biochem Biophys Res Commun 1999; 256: 527-31.

15 Roberts RA, Ganey PE, Ju C, Kamendulis LM, Rusyn I, Klaunig JE. Role of the Kupffer cell in mediating hepatic toxicity and carcinogenesis. Toxicol Sci 2007; 96: 2-15.

16 Yang $\mathrm{H}$, Guo H, Fan K, Zhang B, Zhao L, Hou S, et al. Clearance of Propionibacterium acnes by kupffer cells is regulated by osteopontin through modulating the expression of p47phox. Mol Immunol 2011; 48: 2019-26.

17 Adams DH, Ju C, Ramaiah SK, Uetrecht J, Jaeschke H. Mechanisms of immune-mediated liver injury. Toxicol Sci 2010; 115: 307-21.

18 Welch KD, Reilly TP, Bourdi M, Hays T, Pise-Masison CA, Radonovich $\mathrm{MF}$, et al. Genomic identification of potential risk factors during acetaminophen-induced liver disease in susceptible and resistant strains of mice. Chem Res Toxicol 2006; 19: 223-33.

19 Hinson JA, Roberts DW, James LP. Mechanisms of acetaminopheninduced liver necrosis. Handb Exp Pharmacol 2010; (196): 369-405.

20 Laskin DL, Gardner CR, Price VF, Jollow DJ. Modulation of macrophage functioning abrogates the acute hepatotoxicity of acetaminophen. Hepatology 1995; 21: 1045-50. 
21 Michael SL, Pumford NR, Mayeux PR, Niesman MR, Hinson JA. Pretreatment of mice with macrophage inactivators decreases acetaminophen hepatotoxicity and the formation of reactive oxygen and nitrogen species. Hepatology 1999; 30: 186-95.

22 Jaeschke $\mathrm{H}$. Role of inflammation in the mechanism of acetaminophen-induced hepatotoxicity. Expert Opin Drug Metab Toxicol 2005; 1: 389-97.

23 Ramaiah SK, Rittling S. Pathophysiological role of osteopontin in hepatic inflammation, toxicity, and cancer. Toxicol Sci 2008; 103: 4-13.

24 Rittling SR. Osteopontin in macrophage function. Exp Rev Mol Med 2011; 13: e15.

25 Liu ZX, Han D, Gunawan B, Kaplowitz N. Neutrophil depletion protects against murine acetaminophen hepatotoxicity. Hepatology 2006; 43: 1220-30.

26 Fan K, Dai J, Wang H, Wei H, Cao Z, Hou S, et al. Treatment of collagen-induced arthritis with an anti-osteopontin monoclonal antibody through promotion of apoptosis of both murine and human activated T cells. Arthritis Rheum 2008; 58: 2041-52.

27 Chen Q, Kon J, Ooe H, Sasaki K, Mitaka T. Selective proliferation of rat hepatocyte progenitor cells in serum-free culture. Nat Protoc 2007; 2: 1197-205.

28 Holt MP, Cheng L, Ju C. Identification and characterization of infiltrating macrophages in acetaminophen-induced liver injury. J Leukoc Biol 2008; 84: 1410-21.

29 Han D, Shinohara M, Ybanez MD, Saberi B, Kaplowitz N. Signal transduction pathways involved in drug-induced liver injury. Handb Exp Pharmacol 2010; (196): 267-310.

30 Martin-Murphy BV, Holt MP, Ju C. The role of damage associated molecular pattern molecules in acetaminophen-induced liver injury in mice. Toxicol Lett 2010; 192: 387-94.

31 Fan K, Zhang B, Yang H, Wang H, Tan M, Hou S, et al. A humanized anti-osteopontin antibody protects from Concanavalin A induced-liver injury in mice. Eur J Pharmacol 2011; 657: 144-51.

32 Sahai A, Pan X, Paul R, Malladi P, Kohli R, Whitington PF. Roles of phosphatidylinositol 3-kinase and osteopontin in steatosis and aminotransferase release by hepatocytes treated with methioninecholine-deficient medium. Am J Physiol Gastrointest Liver Physiol 2006; 291: G55-62.

33 Atul Sahai PM, Melin-Aldana H, Green RM, Whitington PF. Upregulation of osteopontin expression is involved in the development of nonalcoholic steatohepatitis in a dietary murine model. Am J Physiol Gastrointest Liver Physiol 2004; 287: G264-73.

34 Cantor H, Shinohara ML. Regulation of T-helper-cell lineage development by osteopontin: the inside story. Nat Rev 2009; 9: 137-41.

35 Gujral JS, Knight TR, Farhood A, Bajt ML, Jaeschke H. Mode of cell death after acetaminophen overdose in mice: apoptosis or oncotic necrosis? Toxicol Sci 2002; 67: 322-8.
36 Agarwal R, Hennings L, Rafferty TM, Letzig LG, McCullough S, James $\mathrm{LP}$, et al. Acetaminophen-induced hepatotoxicity and protein nitration in neuronal nitric-oxide synthase knockout mice. J Pharmacol Exp Ther 2012; 340: 134-42.

37 Michael SL, Mayeux PR, Bucci TJ, Warbritton AR, Irwin LK, Pumford $\mathrm{NR}$, et al. Acetaminophen-induced hepatotoxicity in mice lacking inducible nitric oxide synthase activity. Nitric Oxide 2001; 5: 432-41.

38 Sakurai T, He G, Matsuzawa A, Yu GY, Maeda S, Hardiman G, et al. Hepatocyte necrosis induced by oxidative stress and IL-1 alpha release mediate carcinogen-induced compensatory proliferation and liver tumorigenesis. Cancer Cell 2008; 14: 156-65.

39 Ishida Y, Kondo T, Tsuneyama K, Lu P, Takayasu T, Mukaida N. The pathogenic roles of tumor necrosis factor receptor p55 in acetaminophen-induced liver injury in mice. J Leukoc Biol 2004; 75: 59-67.

40 Schwabe RF, Brenner DA. Mechanisms of liver injury. I. TNF-alphainduced liver injury: role of IKK, JNK, and ROS pathways. Am J Physiol Gastrointest Liver Physiol 2006; 290: G583-9.

41 Gardner CR, Laskin JD, Dambach DM, Chiu H, Durham SK, Zhou P, et al. Exaggerated hepatotoxicity of acetaminophen in mice lacking tumor necrosis factor receptor-1. Potential role of inflammatory mediators. Toxicol Appl Pharmacol 2003; 192: 119-30.

42 Ishibe T, Kimura A, Ishida Y, Takayasu T, Hayashi T, Tsuneyama K, et al. Reduced acetaminophen-induced liver injury in mice by genetic disruption of IL-1 receptor antagonist. Lab Invest 2009; 89: 68-79.

43 Blazka ME, Elwell MR, Holladay SD, Wilson RE, Luster MI. Histopathology of acetaminophen-induced liver changes: role of interleukin 1 alpha and tumor necrosis factor alpha. Toxicol Pathol 1996; 24 : 181-9.

44 James LP, Lamps LW, McCullough S, Hinson JA. Interleukin 6 and hepatocyte regeneration in acetaminophen toxicity in the mouse. Biochem Biophys Res Commun 2003; 309: 857-63.

45 Ramaiah SK, Jaeschke $\mathrm{H}$. Role of neutrophils in the pathogenesis of acute inflammatory liver injury. Toxicol Pathol 2007; 35: 757-66.

46 Koh A, da Silva AP, Bansal AK, Bansal M, Sun C, Lee H, et al. Role of osteopontin in neutrophil function. Immunology 2007; 122: 466-75.

47 Christensen B, Kazanecki CC, Petersen TE, Rittling SR, Denhardt DT, Sorensen ES. Cell type-specific post-translational modifications of mouse osteopontin are associated with different adhesive properties. J Biol Chem 2007; 282: 19463-72.

48 Kazanecki CC, Uzwiak DJ, Denhardt DT. Control of osteopontin signaling and function by post-translational phosphorylation and protein folding. J Cell Biochem 2007; 102: 912-24.

$49 \mathrm{Kim} \mathrm{HJ}$, Lee HJ, Jun JI, Oh Y, Choi SG, Kim H, et al. Intracellular cleavage of osteopontin by caspase- 8 modulates hypoxia/reoxygenation cell death through p53. Proc Natl Acad Sci U S A 2009; 106: 15326-31. 